\title{
Effekte einer einmaligen oralen Eisenapplikation nach der Geburt auf die Gewichtsentwicklung sowie auf hämatologische Variablen, Hämoglobinderivate und Blutgasgehalte der Milchrindkälber wäh- rend der Aufzucht in Gruppenhaltung mit Tränkeautomatenfütte- rung
}

\begin{abstract}
Title of the paper: Effects of a single oral iron application on growth and on hematological variables, hemoglobin derivatives and blood gas content in group housed feeder-fed dairy calves

To characterize physiological variables in dairy calves of different developmental quality and fitness (hemoglobin content of blood low HbG1, moderate HbG2 and high HbG3) at early gowth with uniform husbandry system on dairy calves (71 German Holstein Friesian, 6 German Red Pied, 36 male and 41 female) measurements were done before (5 days of age) and repeatedly at 15, 40 and 60 days of age when calves were kept in groups with automatic feeding. Mean birth weight was not different between groups. Growth performance of calves of HbG1 was smaller then that of calves in HbG2 and HbG3. Correlations of birth weight and body weight at the following testing points with the growth criteria were of different degree within the groups. Hematological variables of the calves within the three groups changed with growth in different ways. Significant mean value differences between groups could be found for $\mathrm{Hb}, \mathrm{O}_{2} \mathrm{CAP}, \mathrm{O}_{2} \mathrm{CONT}$, MetHb up to 40 days, for $\mathrm{Hk}, \mathrm{HHb}$, and for $\mathrm{O}_{2} \mathrm{SAT}$ up to 60 days, for MCHC up to 15 days, and for the lactate concentration at 40 days and 60 days of age. The prophylactic single oral iron application of $1000 \mathrm{mg}$ in calves at birth and the iron supply of $100 \mathrm{mg} / \mathrm{kg}$ by milk replacer led to plasmairon concentrations of 20 to $40 \mu \mathrm{mol} / \mathrm{l}$, but the erythropoietic reactions in the calves were different. Relationships between the quality of erythropoiesis and iron access were assessed considering the changes of the physiological variables and the initial situation in calves at birth.
\end{abstract}

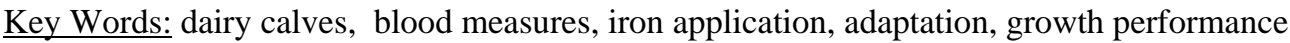

\section{Zusammenfassung}

Um physiologische Variablen von Milchrindkälbern mit einer unterschiedlichen Entwicklungsqualität und Fitness (Hämoglobingehalt des Blutes klein HbG1, mittelmäßig HbG2 und groß HbG3) im Verlaufe der frühen Wachstumsperiode bei einheitlichen Haltungsbedingungen zu charakterisieren, wurden an Kälbern (71 Deutsche Holstein Friesian, 6 Deutsche Rotbunte, 36 männliche, 41 weibliche) vor (5 LT) und während der Gruppenhaltung mit automatischer Fütterung (15, 40 und 60 LT) wiederholt Untersuchungen hämatologischer Messwerte vorgenommen. Das mittlere Geburtsgewicht war zwischen den Gruppen nicht signifikant unterschiedlich. Die Wachstumsleistung der Kälber der HbG1 war kleiner als die von HbG2 und HbG3. Geburtsgewicht und Körpermasse der folgenden Untersuchungspunkte hatten Korrelationen mit den Wachstumskriterien, die in den $\mathrm{Hb}$ Gruppen von unterschiedlicher Stärke waren. Die hämatologischen Variablen änderten sich bei Kälbern der drei Hb-Gruppen in unterschiedlichem Grade während der Aufzucht. Signifikante Mittelwertunterschiede zwischen den Gruppen konnten für $\mathrm{Hb}, \mathrm{O}_{2} \mathrm{CAP}, \mathrm{O}_{2} \mathrm{CONT}$, MetHb bis $40 \mathrm{LT}$, für $\mathrm{Hk}$, $\mathrm{HHb}$ und für $\mathrm{O}_{2} \mathrm{SAT}$ bis $60 \mathrm{LT}$, für MCHC bis 15 LT und für Laktat bei 40 und 60 LT nachgewiesen werden. Die prophylaktische orale Eisenapplikation von 1000 mg nach der Geburt und die durch den Milchaustauscher verfügbare Fe-Menge von 100 mg/kg hatten Plasmaeisenwerte zwischen 20 und $40 \mu \mathrm{mol} / \mathrm{l}$ zur Folge, die erythropoetischen Reaktionen waren bei den Kälbern unterschiedlich. Zusammenhänge zwischen der Qualität der Erythropoese und der Eisenverfügbarkeit werden in Verbindung mit der Änderung physiologischer Variablen und der Ausgangssituation bei den Kälbern nach der Geburt eingeschätzt. 
1. Einleitung

Die Entwicklungsqualität der Kälber, gemessen an der Variation physiologischer Variablen, ist in Verbindung mit direkten und indirekten Verlusten während der Aufzucht untersucht worden (WILSON et al., 2000). Unter Bedingungen einer standardisierten Ernährung und einer vorübergehenden räumlich und zeitlich begrenzten Nahrungsverfügbarkeit der Tiere, wie bei den in wechselnder Gruppenzusammensetzung am Futterautomaten aufgezogenen Milchrindkälbern, wirkte sich die Entwicklungsqualität und physische Fitness der Kälber auf die Wachstumsleistung, die Herzfrequenz und das Aktivitätsniveau der Tiere aus und beeinflusste auch die Rhythmik (STEINHARDT und THIELSCHER, 2002). In der vorliegenden Arbeit wurde die Anpassung der Kälber mit einem unterschiedlichen Hämoglobingehalt des Blutes (Hb) im Alter von 5 Lebenstagen (LT) unter einheitlichen Aufzuchtbedingungen charakterisiert und die Effektivität einer prophylaktischen oralen Eisenapplikation nach der Geburt eingeschätzt. Die folgenden Fragen waren von Interesse: (1) Wie ändern sich physiologische Variablen der Kälber mit unterschiedlicher Entwicklungsqualität nach der Geburt im Verlaufe von Wachstum und Adaptation der Tiere unter einheitlichen Aufzuchtbedingungen? (2) Wie ist die Wirksamkeit einer oralen Eisenapplikation bei diesen Kälbern hinsichtlich der hämatologischen Variablen und der Gewichtszunahmen der Tiere?

2.. Material und Methodik

Untersuchte Tiere, Haltungsbedingungen: Kälber der Milchrindherde des Institutes für Tierzucht und Tierverhalten (71 Deutsche Holstein Friesian, DHF; 6 Deutsche Rotbunte, DRB; 36 männliche und 41 weibliche) der Kalbeperiode 1997/1998 wurden untersucht. Nach der Geburt blieben die Kälber bis zum Alter von 5 bis 7 Lebenstagen (LT) in einer Gruppenbox mit Stroheinstreu im Abkalbebereich des Stallgebäudes (Laufstall), in welchem die Milchrinder gehalten wurden. Die Kälber erhielten mehrmals Kolostrum der Mutter (07.00 Uhr und 16.00 Uhr, 1 bis 2 l pro Angebot), danach gepoolte Frischmilch aus einem Eimer mit Nuckel. Die Tiere erhielten nach ausreichender Kolostrumversorgung einmalig als Eisenapplikation $5 \mathrm{ml}$ Myofer 200 (Hoechst) pro Tier über die Milch verabreicht. In Gruppenhaltung (2,5 $\mathrm{m}^{2}$ pro Tier bei voller Besetzung) mit Tränkeautomatenfütterung (Tränkeautomat der Fa. Förster, Festzeitsystem, Tränkeschema: 1 Woche 6 l; 1 Woche $6 \mathrm{l}$ auf $8 \mathrm{l}$ ansteigend; 4 Wochen 8 l; 6 Wochen $8 \mathrm{l}$ auf $2 \mathrm{l}$ abfallend; 1 Woche $2 \mathrm{l}$ pro Tag Milchaustauscher (MAT) Färsenstart S von Denkavit Futtermittel GmbH, 125 g pro Liter Wasser) verblieben die Kälber 6 Wochen als Einstallungsgruppe und danach 6 Wochen in einer benachbarten Box als Ausstallungsgruppe. Die MAT-Verfügbarkeit war auf 4 Zeitpunkte pro Tag (03.00 Uhr, 08.00 Uhr, 15.00 Uhr und 20.00 Uhr) beschränkt, pro Abruf konnten 2 l MAT erreicht werden. Die Sperrzeit nach einem Abruf betrug 120 min. Die Tränkestation war in der Nacht beleuchtet. Die Fenster des Aufzuchtstalles waren mit Windschutznetzen versehen. Pelletiertes Kraftfutter konnte über einen Automaten ansteigend bis maximal $3 \mathrm{~kg}$ pro Tier und Tag abgerufen werden. Gleichzeitig wurden Heu und Silage angeboten, und es bestand die Möglichkeit der Wasseraufnahme aus Selbsttränken und der Kochsalzaufnahme an Lecksteinen. Wägungen der Kälber wurden an spezifischen Alterspunkten wie Geburt, Einstallung in die Gruppen- 
haltung, Umstallung in eine andere Gruppenhaltung und Ausstallung vorgenommen. Aus diesen Messungen ist die Wachstumsrate der Kälber errechnet worden.

Untersuchungsablauf, Messungen: Bei einem Alter der Kälber von 5, 15, 40 und 60 LT erfolgten Tierwägungen sowie Messungen der Körpertemperatur (RT) und Blutuntersuchungen. Mit Hilfe der zugebildeten Körpermasse und des Alters wurde die Wachstumsrate (tägliche Zunahme) ermittelt. Die Körpertemperatur wurde mit gebräuchlichen Fieberthermometern festgestellt. Zur Blutentnahme waren die Kälber mit einem Strickhalfter fixiert worden. In peripheren venösen Blutproben (V. jugularis) wurden der Hämatokritwert (Hk), die Hämoglobinkonzentration (Hb), Hämoglobinderivate und -varianten, Gasgehalte und -drucke ermittelt. Laktat konnte im Analysenautomaten (Kone, Finnland) mit Reagenzien der Firma Boehringer bestimmt werden. Die Blutproben wurden mit dem AVL 912 CO-Oxylite von Medical Instruments AG, mit welchem neben Messgrößen des Säure-Basen-Status die Hämoglobinkonzentration, Sauerstoffsättigung $\left(\mathrm{O}_{2} \mathrm{SAT}\right)$, Sauerstoffkapazität $\left(\mathrm{O}_{2} \mathrm{CAP}\right)$ und der Sauerstoffgehalt $\left(\mathrm{O}_{2} \mathrm{CONT}\right)$, die Hämoglobinderivate Oxyhämoglobin $\left(\mathrm{O}_{2} \mathrm{Hb}\right)$, Desoxyhämoglobin ( $\mathrm{HHb})$, Carboxyhämoglobin (COHb), Methämoglobin (MetHb) und Sulfhämoglobin (SHb) ermittelt werden können, untersucht. Hk wurde mit der Mikrohämatokritmethode bestimmt.

Bearbeitung der Ergebnisse, statistische Methoden: Mit Hilfe der Hämoglobinkonzentration des Blutes ( $\mathrm{Hb}$ ) der Kälber bei 5 LT ist eine Klassifizierung und eine Zuordnung der Tiere zu folgenden Hb-Gruppen vorgenommen worden: HbG1 = Hb <9,2 g/dl; HbG2 = Hb 9,2 bis 10,9 g/dl und HbG3 = Hb > $11 \mathrm{~g} / \mathrm{dl}$. Die Auswertung fand unter folgenden Gesichtspunkten statt: (1) Prüfung der Variablen zwischen den Kälbergruppen an den Alterspunkten, (2) Prüfung der Variablen zwischen den Alterspunkten in den Kälbergruppen, (3) Prüfung der Korrelationen von Variablen zwischen den Alterspunkten und (4) Prüfung der Korrelationen zwischen den Variablen an den Alterspunkten in den Kälbergruppen. Die Bearbeitung der Ergebnisse wurde mit PC-Statistik von Topsoft Hannover, mit Systat sowie Sigma Plot von SPSS Science Software vorgenommen (Korrelations- und Regressionsrechnung, ANOVA und ANOVA for repeated measures). Mittelwertprüfungen zweier Gruppen wurden mit dem t-Test und Wilcoxon-Test vorgenommen. Die Irrtumswahrscheinlichkeiten (Bonferroni adjusted probability) sind in den Tabellen und Abbildungen angegeben und, wenn nicht ausdrücklich erwähnt, mit 5 \% angenommen worden.

\section{3. $\quad$ Ergebnisse}

\section{Körpermasse, Beziehungen der Wachstumskriterien}

Die Wachstumsraten und die Körpermassen der Kälbergruppen waren unterschiedlich, wie in einem vorangegangenen Untersuchungsbericht bereits dargestellt worden ist (STEINHARDT und THIELSCHER, 2002). Das Geburtsgewicht wies mit der Körpermasse an den folgenden Messpunkten Korrelationen auf, die bei Tieren der HbG2 und HbG1 enger waren als bei jenen der HbG3 (Tab.). Die KM hatte in den folgenden Altersperioden positive Korrelationen mit den Wachstumskriterien, deren Grad zwischen den Hb-Gruppen unterschiedlich, bei Kälbern der HbG3 meistens stärker war (Tab.). Bei Kälbern der HbG3 konnten schwache negative Beziehungen zwischen Hb bei 7 LT und DIKM bei 7 LT $(r=-0,418, p=0,0337)$ sowie zwischen Hb bei 15 LT 
und DIKM bei 7 LT $(r=-0,357, p=0,04)$ und Hb bei 15 LT und Wachstumsrate bei 7 LT ( $r=-0,357, p=0,0399)$ festgestellt werden.

Tabelle

Korrelationen der Körpermasse (KM) bei der Geburt und bei verschiedenen Alterspunkten mit der Wachstumsrate (WR) der Milchrindkälber in den Gruppen nach dem Hämoglobingehalt des Blutes an verschiedenen Alterspunkten (Correlations of body weight at birth and at different age points with growth rate (WR) in dairy calves with different hemoglobin content of blood)

\begin{tabular}{|c|c|c|c|c|c|}
\hline & & $\begin{array}{l}\text { KM } \\
\text { Geburt } \\
\end{array}$ & $\begin{array}{l}\text { KM } \\
\text { 7LT } \\
\end{array}$ & $\begin{array}{l}\text { KM } \\
\text { 50LT }\end{array}$ & $\begin{array}{l}\text { KM } \\
78 \mathrm{LT} \\
\end{array}$ \\
\hline KM 7 LT & HbG1 & $0,895^{* *}$ & & & \\
\hline \multirow[t]{2}{*}{ (kg) } & HbG2 & $0,803^{* *}$ & & & \\
\hline & HbG3 & $0,662 * *$ & & & \\
\hline \multirow{3}{*}{$\begin{array}{l}\text { WR } 7 \text { LT } \\
(\mathrm{kg} / \mathrm{d})\end{array}$} & HbG1 & $-0,034$ & 0,398 & & \\
\hline & HbG2 & $-0467^{*}$ & 0,126 & & \\
\hline & HbG3 & $-0,312$ & $0,491 * *$ & & \\
\hline \multirow{3}{*}{$\begin{array}{l}\text { KM } 50 \mathrm{LT} \\
(\mathrm{kg})\end{array}$} & HbG1 & $0,789 * *$ & $0,817^{* *}$ & & \\
\hline & HbG2 & $0,710^{* *}$ & $0,782 * *$ & & \\
\hline & HbG3 & $0,431^{* *}$ & $0,629 * *$ & & \\
\hline \multirow{3}{*}{$\begin{array}{l}\text { WR } 50 \text { LT } \\
(\mathrm{kg} / \mathrm{d})\end{array}$} & HbG1 & $-0,035$ & 0,005 & $0,549^{*}$ & \\
\hline & HbG2 & 0,085 & 0,212 & 0,730 ** & \\
\hline & HbG3 & $-0,003$ & $0,338^{* *}$ & $0,886^{* *}$ & \\
\hline \multirow{3}{*}{$\begin{array}{l}\text { KM } 78 \text { LT } \\
(\mathrm{kg})\end{array}$} & HbG1 & $0,660^{* *}$ & $0,791 * *$ & $0,875^{* *}$ & \\
\hline & HbG2 & $0,515^{* *}$ & $0,600 * *$ & $0,574 * *$ & \\
\hline & HbG3 & $0,399^{*}$ & $0,595 * *$ & $0,910^{* *}$ & \\
\hline \multirow{3}{*}{$\begin{array}{l}\text { WR } 78 \text { LT } \\
\text { (kg/d) }\end{array}$} & HbG1 & 0,161 & 0,382 & $0,567 * *$ & $0,825^{* *}$ \\
\hline & HbG2 & 0,061 & 0,283 & 0,246 & $0,865^{* *}$ \\
\hline & HbG3 & 0,064 & $0,377^{*}$ & $0,803^{* *}$ & $0,926 * *$ \\
\hline
\end{tabular}

$* \mathrm{p}<0,05 ; * * \mathrm{p}<0,01 ;$ LT $=$ Lebenstage
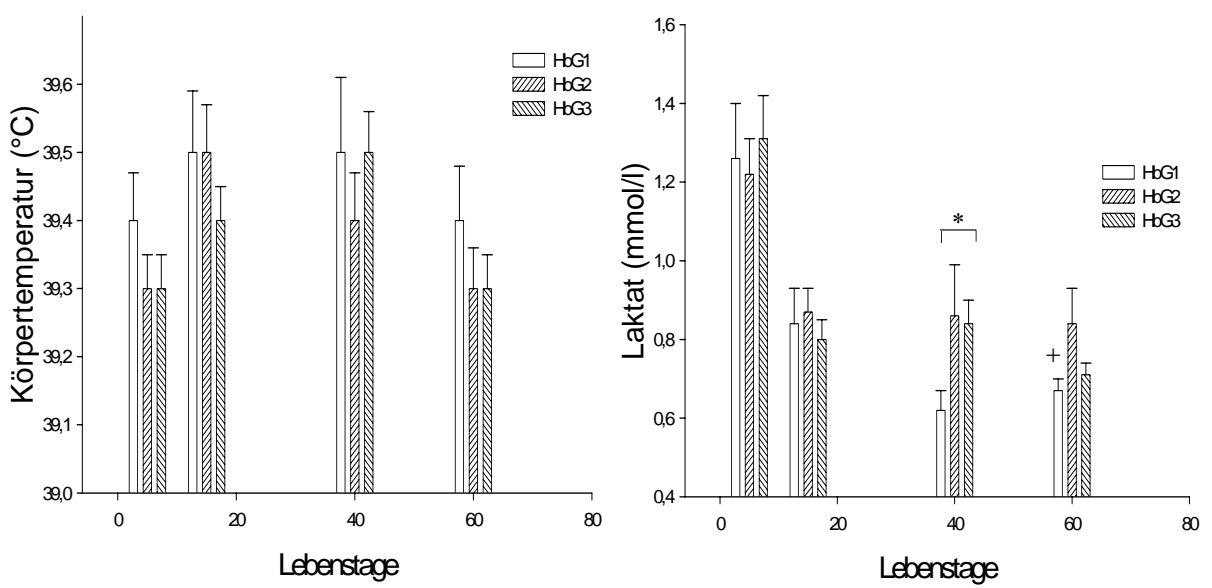

Abb. 1: Körpertemperatur und Laktatkonzentration des Blutplasmas bei Milchrindkälbern an verschiedenen Alterspunkten, LSM und SEM (Body temperature and lactate concentration of blood plasma in dairy calves at different age points, LSM and SEM)

\section{Körpertemperatur, Laktat}

Die Körpertemperatur war bei 15 und 40 LT höher als an den anderen Alterspunkten (Abb. 1). Die Änderungen der Körpertemperatur gingen bei den Hb-Gruppen an den Alterspunkten in unterschiedlicher Weise vonstatten. Mittelwertunterschiede derselben zwischen den Hb-Gruppen und zwischen den Alterspunkten waren nicht signifikant. Die Laktatkonzentration war bei 5 LT signifikant größer als an den übrigen Alterspunkten (Abb. 1). Signifikante Unterschiede der Mittelwerte zwischen den Hb-Gruppen waren bei 40 und $60 \mathrm{LT}$ festzustellen. 


\section{Hämatologische Variablen, Sauerstoffgehalt des Blutes, Plasmaeisen}

$\mathrm{Hb}$ und Hk waren zwischen den Gruppen bis zum Alter von 40 LT, Hk war bei 60 LT noch zwischen HbG1 und HbG3 signifikant verschieden (Abb. 2). Die Änderungen von $\mathrm{Hb}$ und $\mathrm{Hk}$ der Kälber in den Hb-Gruppen waren an den Alterspunkten unterschiedlich, und die Mittelwerte waren zwischen den Alterspunkten signifikant verschieden (Abb. 2). $\mathrm{O}_{2}$ CAP wies weitgehend die gleichen Unterschiede und Änderungen wie $\mathrm{Hb}$ auf (Ergebnisse nicht weiter angeführt). MCHC war bei $5 \mathrm{LT}$ zwischen den Gruppen signifikant verschieden (Abb. 2). Mit zunehmendem Alter der Kälber wurde MCHC größer. Die Mittelwerte von MCHC zwischen den Alterspunkten waren signifikant unterschiedlich. Plasmaeisen der Kälber aus HbG2 war bei 60 LT signifikant größer als bei jenen aus HbG1 und HbG3. Zwischen den Alterspunkten war es bei Kälbern der HbG3 signifikant unterschiedlich. $\mathrm{O}_{2} \mathrm{CONT}$ des venösen Blutes änderte sich mit dem Alter der Kälber signifikant und war bei Tieren der HbG3 an allen Alterspunkten größer als bei jenen der HbG1 und HbG2. Bis zum Alter von $60 \mathrm{LT}$ waren die Mittelwertunterschiede von $\mathrm{O}_{2} \mathrm{CONT}$ zwischen den Gruppen signifikant (Abb. 3). Bei 5 LT und bei $60 \mathrm{LT}$ wiesen Kälber der HbG1 die größte $\mathrm{O}_{2} \mathrm{SAT}$ des Blutes auf. Sie war bei Kälbern der HbG1 im Alter von 15 und 40 LT kleiner und bei jenen der HbG2 und HbG3 in diesem Alter größer als bei 5 und 60 LT. Signifikante Mittelwertunterschiede von $\mathrm{O}_{2} \mathrm{SAT}$ zwischen den Alterspunkten ließen sich nur bei Kälbern der HbG2 nachweisen (Abb. 3). Kälber der HbG1 wiesen den größten COHbAnteil des Blutes auf (Abb. 3). Signifikante Unterschiede des mittleren COHb-Anteils waren bei 5 LT sowie bei 40 und 60 LT festzustellen. Bei Kälbern der HbG1 wurde der COHb-Anteil des Blutes mit zunehmendem Alter der Tiere kleiner und bei jenen der HbG2 und HbG3 bis zum Alter von 40 LT größer, dann bis 60 LT wieder kleiner.
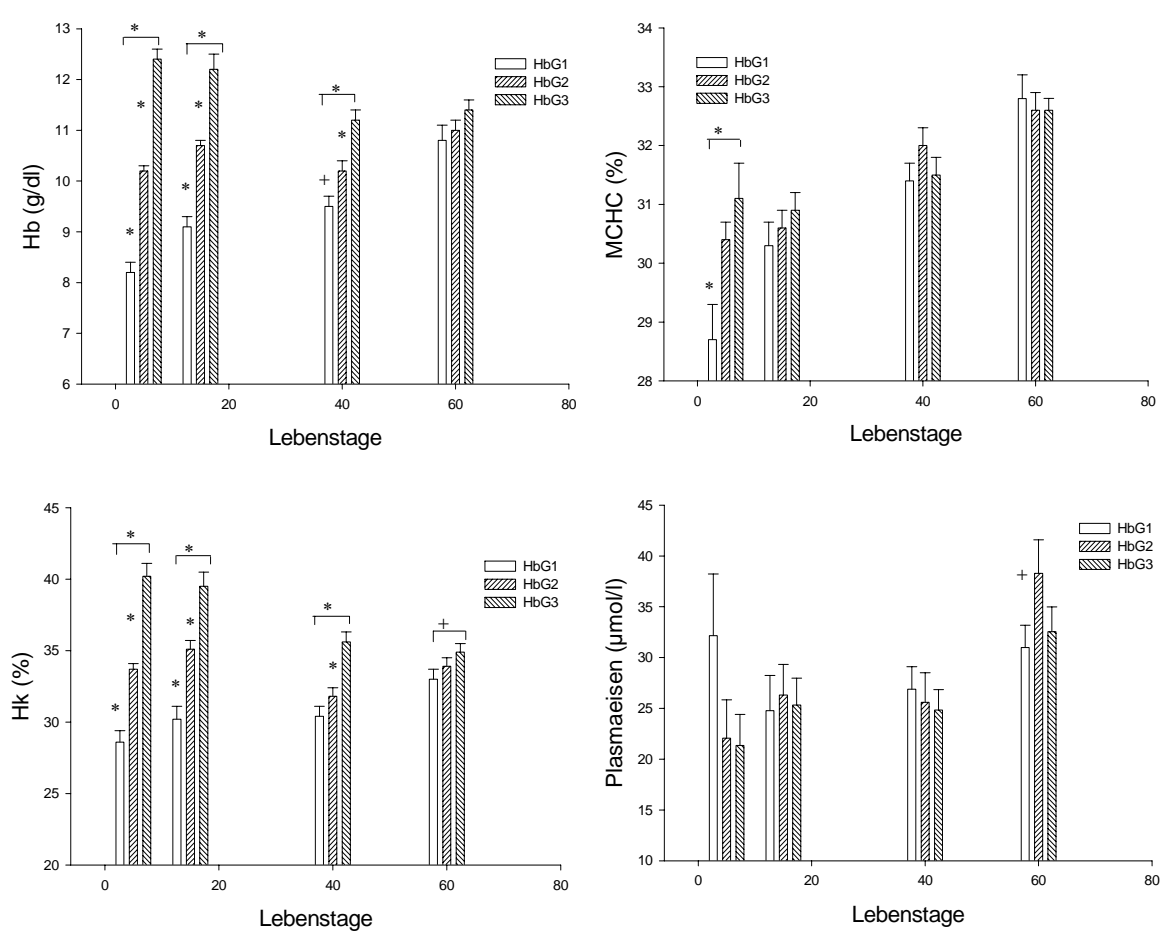

Abb. 2: Hämoglobinkonzentration, Hämatokrit, mittlere korpuskuläre Hämoglobinkonzentration (MCHC) und Plasmaeisenkonzentration bei Milchrindkälbern an verschiedenen Alterspunkten, LSM und SEM (Hemoglobin content, hematocrite, mean corpuscular hemoglobin concentration and plasmairon concentration in dairy calves at different age points, LSM and SEM) 

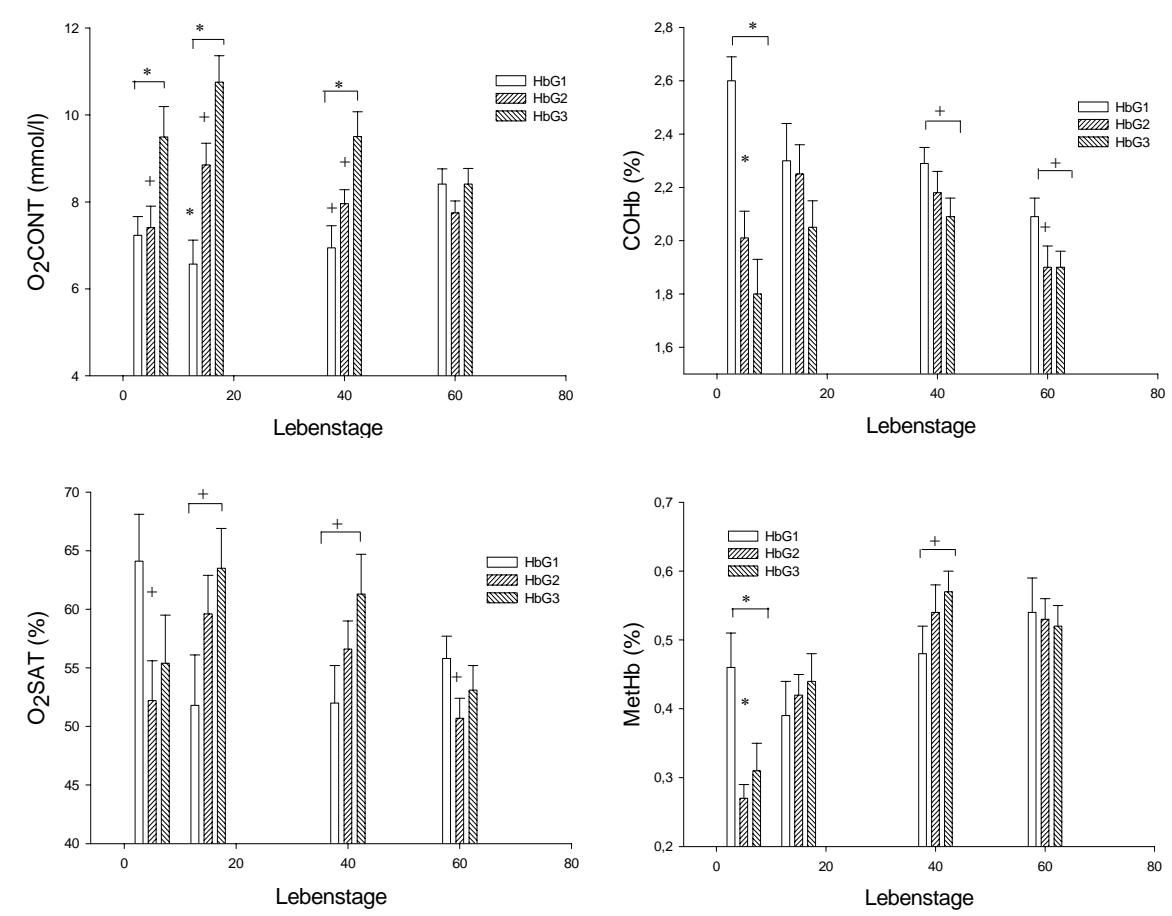

Abb. 3: Sauerstoffgehalt (O2CONT), Sauerstoffsättigung (O2SAT), Carboxyhämoglobinanteil (COHb) und Methämoglobinanteil (MetHb) des Blutes der Milchrindkälber an verschiedenen Alterspunkten, LSM und SEM (Oxygen content, oxygen saturation, carboxyhemoglobin fraction and methemoglobin fraction of blood in dairy calves at different age points, LSM and SEM)

\section{4.}

\section{Diskussion}

Die unterschiedlichen Änderungen der physiologischen Variablen bei den Kälbern der drei Hb-Gruppen unter weitgehend einheitlichen Haltungsbedingungen (Abb. 1 bis 3), die hohen Korrelationen von Geburtsgewicht und Körpermasse bei Kälbern der HbG1 und HbG2 und die unterschiedliche Wachstumsleistung der Tiere (Tab.) weisen auf spezifische Effekte der genetischen und der Umweltfaktoren bei Kälbern mit unterschiedlicher Entwicklungsqualität nach der Geburt hin. Die Körpertemperatur lag infolge des intensiveren Stoffwechsels und der Anpassung der Wärmeregulation bei 15 LT und 40 LT höher als bei 5 und 60 LT (Abb. 1). Kälber der drei Hb-Gruppen wiesen unterschiedliche Aktivitäts- und Ruhezeiten sowie auch unterschiedliche rhythmische Änderungen derselben und der Herzfrequenz im Verlaufe des Tages auf, die sich während der Aufzucht änderten (STEINHARDT und THIELSCHER, 2002). Die variierende $\mathrm{O}_{2}$-Transportkapazität des Blutes der Kälber, die durch die Hämoglobinkonzentration und die Hämoglobinvariante (HbF, $\mathrm{HbA}, \mathrm{HbB}$ ) beeinflusst wird (CAMBIER et al., 2000; GUSTIN et al., 1997), wurde in den ersten Lebenswochen durch die Herzfrequenz und die Sauerstoffextraktion in den Geweben kompensiert (Abb. 2 und 3), denn die Laktatkonzentration war zwischen den Gruppen nicht unterschiedlich (Abb. 1). Signifikante Mittelwertunterschiede der Laktatkonzentration zwischen den HbGruppen bei 40 und 60 LT waren auf die unterschiedliche Reaktivität der Kälber in diesem Alter zurückzuführen. Verkleinerungen von $\mathrm{Hk}$ und $\mathrm{Hb}$ in den ersten Lebensstunden und -tagen (ADAMS et al., 1992, 1993; BOSTEDT et al., 1990; KNOWLES et al., 2000; KÜHNE et al., 2000; KURZ und WILLETT, 1991), eine Folge der Erweiterung des Gefäßsystems und der damit verbundenen Blutvolumenvergrößerung (vorwiegend Plasmavolumen, Hämodilution), gehen konform mit weiteren postnatalen 
Anpassungen, die periphere und zentrale chemorezeptive und hormonelle Regulationsmechanismen und auch die Plasma-Fe-Konzentration betreffen, die in den ersten Lebenstagen bei Kälbern abfällt (KNOWLES et al., 2000; MIYATA et al., 1984). In Abhängigkeit von der Entwicklungsqualität und Anpassung des Tieres an die intrauterinen Lebensbedingungen und unter dem Einfluss des Geburtsverlaufes (z B. gestörte placento-fetale Transfusion) erfolgen die Änderungen physiologischer Variablen in unterschiedlichem Grade (STEINHARDT und THIELSCHER, 2000). Beträchtlich variierende $\mathrm{Hb}$ und $\mathrm{Hk}$, höhere Grade der $\mathrm{O}_{2}$-Sättigung und größere Anteile an $\mathrm{COHb}$, infolge der Interferenz indirekt ein Ausdruck des HbF-Anteils, und an MetHb der Kälber in HbG1 bei 5 LT (Abb. 2 und 3) sprechen dafür, dass bei diesen Tieren die Erythropoese vor der Geburt bereits stärker als bei den Kälbern von HbG2 und HbG3 in der Qualität beeinflusst war. Erythropoietin (EPO) wird bei Feten in der Leber und bei Arten mit langer intrauteriner Entwicklungsperiode im geburtsnahen Zeitraum zunehmend in der Niere gebildet. Die Expression von EPO im glomerulären Gewebe bei Neugeborenen wird durch hohe Cortisolkonzentrationen gehemmt (Schaf: LIM et al., 1996). Ein zeitspezifischer Effekt der Glukokortikoidwirkung bei der Umschaltung der Produktion von fetalem auf diejenige von adultem Hämoglobin ist nachgewiesen worden (Schaf: WINTOUR et al., 1985). Hierzu wären weitere Untersuchungen an Kälbern notwendig.

Hinsichtlich der Nahrungs-, speziell auch der Spurenelementaufnahme der Kälber und des Flüssigkeitsbestandes in den ersten Lebenstagen sind mehrere Faktoren wirksam, die zur Variation der untersuchten Variablen beigetragen haben können. Die von den Tieren aufgenommenen Mengen an Kolostrum und Milch waren interindividuell sehr unterschiedlich. Das Alter der Kuh (Laktationsnummer) beeinflusste die Fe-Konzentration im Kolostrum nicht (KUME und TANABE, 1993), die Lactoferrinkonzentration war bei multiparen Kühen größer als bei Primiparen (TSUJI et al., 1990). Lactoferrin kann die Fe-Absorption aus dem Darm fördern, es stabilisiert Fe2+ im Gastroinstinaltrakt oder Blutplasma unmittelbar nach der Verabreichung und beschleunigt die Aufnahme des Fe in Hämoglobin (NAGASAKO et al., 1993; KUME und TANABE, 1996). Eisen ist ein essentielles Element und dient in Hämoglobin, Myoglobin und Cytochrom C zur Bereitstellung zellulärer Energie, in Cytochrom P450 und in der Ribonukleotidreduktase ist es am Xenobiotikametabolismus und an der DNA-Synthese beteiligt. Spezielle Resorptionsmechanismen für Fe im proximalen Dünndarm sind energieabhängig, sättigbar und von einer intakten Proteinsynthese abhängig. Die Anpassung der Eisenresorption scheint durch bedarfsgerechte Expression von Eisentransportproteinen in der Bürstensaummembran des Duodenum (Divalent cation transporter; DCT1, iron regulatory protein, IRP) zu erfolgen (SCHÜMANN und HUNDER, 1998). Über die entwicklungsphysiologische Ausprägung dieser Mechanismen der Kälber sind keine Untersuchungsbefunde vorhanden.

Prinzipiell kann die Fe-Verfügbarkeit durch die Fe-Reserven zum Zeitpunkt der Geburt, durch Geschlecht, Wachstumsrate, chemische Form des verabreichten Fe, Zusammensetzung der Nahrung, Malabsorption, Grad der intermediären Fe-Utilisierung und durch Fe-Verluste des Körpers modifiziert werden. Die in der Leber als Hauptspeicherorgan vorhandene Fe-Menge variierte bei neugeborenen Kälbern sowohl von Färsen als auch von Kühen beträchtlich (MILTENBURG et al., 1991; STEINHARDT et al. 1993). Kommerzielles gepooltes fetales Rinderserum enthielt 1,8 bis 2,2 mg/ml Transferrin, dessen Sättigung zwischen 55 und 92\% variierte (KAKUTA et al., 1997). 
Zwischen Ferritin und der Transferrinsättigung bestand eine enge Korrelation ( $\mathrm{r}=$ 0,886). Dies lässt die individuell unterschiedliche Nutzung des Speichers und auch die unterschiedliche Entwicklung des Organs (Größenzunahme des Speichers) selbst erkennen. Infolge des Geburtsverlaufes können hohe Anteile der fetalen Erythrozytenmenge in den Eihäuten verbleiben, so dass den Neugeborenen eine beträchtliche Menge an Fe nicht mehr zur Verfügung steht. Die prophylaktische Fe-Behandlung und die durch den Milchaustauscher verfügbare Fe-Menge $(100 \mathrm{mg} / \mathrm{kg})$ hatten Plasmaeisenkonzentrationen zur Folge, die im Mittel zwischen 20 und $40 \mu \mathrm{mol} / \mathrm{l}$ lagen (Abb. 2). Die Änderungen von $\mathrm{Hb}$ und $\mathrm{Hk}$ deuteten darauf hin (Abb. 2), dass die erythropoetischen Reaktionen in den Kälbergruppen verschieden waren. Mit dem Alter der Kälber zunehmende Mittelwerte von MCHC lassen die stetige Erneuerung der Erythrozytenpopulation erkennen (Abb. 2). Zunehmende Anteile von COHb bei Kälbern der HbG2 und HbG3 bei 15 und 40 LT (Abb. 3) sind ein Ausdruck einer stark gesteigerten Erythropoese, die von einer vermehrten HbF-Bildung begleitet ist. Die vorher genannten Befunde stimmen mit jenen auf der Grundlage von Reagenten-Nichtreagenten-Bewertungen (STEINHARDT und THIELSCHER, 1998; 2000; STEINHARDT et al., 2000) und mit denen von MILTENBURG et al. (1991) überein, in welchen differente Reaktionsweisen bei Kälbern mit hohem bzw. niedrigem Hb-Ausgangswert am Ende der 1. Lebenswoche und dann während des Wachstums nach erhöhter Fe-Zufuhr über den Milchaustauscher festgestellt wurden.

Parenterale Verabreichung von Fe führte bei Dosierungen von 1000 mg i.m. pro Tier zu mittleren Plasma-Fe-Konzentrationen, die über $40 \mu \mathrm{mol} / \mathrm{l}$ und zu mittleren $\mathrm{Hb}$, die zwischen 12,1 und 12,9 g/dl in der folgenden Zeit bis zum Alter von $43 \mathrm{LT}$ lagen (BOSTEDT et al., 2000). Fe- Supplementierungen bei Kälbern in der frühen Aufzuchtperiode betreffen in anderen Untersuchungen $40 \mathrm{mg}$ pro Tier und Tag (KUME und TANABE, 1996) und $50 \mathrm{mg} \mathrm{Fe} / \mathrm{kg}$ Milchaustauscher (LINDT und BLUM, 1994). Die Zielstellungen der Behandlungen der Tiere, die für die Optimierung herangezogenen Kriterien (Entwicklungsqualität, physische Fitness, Krankheitsresistenz, Lebensdauer) und, wie durch die vorliegenden Untersuchungen offensichtlich wurde, auch der Entwicklungszustand der Kälber nach der Geburt sind in diesem Zusammenhang von Interesse. Die vorgenommenen Supplementierungen von Fe waren in vielen Fällen ausreichend, um den Erythropoeseeisenbedarf in Abhängigkeit vom genetischen Material und von den Aufzuchtbedingungen bei einem Anteil der Kälber zu gewährleisten und förderten teilweise auch den Gewebeeisenbedarf (Muskelwachstum, Myoglobin). Die größte Menge Fe, ob oral verabreicht oder injiziert, wird in der frühen Aufzuchtperiode durch die Erythrozyten genutzt, und nur eine kleine Menge wird an den Speicherorten angereichert (MIYATA et al., 1984).

Effekte anderer Faktoren wie Protein-Energie-Ernährung, Wachstumsqualität, Muskelbildung, körperliche Aktivität auf hämatologische Variablen der Kälber während der frühen Aufzuchtperiode sind bisher nicht ausreichend zu quantifizieren. In welchem Maße ein ausreichender Fe-Bestand des Tierkörpers in den ersten Lebenswochen die Aufnahme und Verwertung von Rauhfutter beeinflusst, ist bisher nicht sicher einzuschätzen. Bekanntlich fördert die Festfutteraufnahme erythropoetische Vorgänge und trägt zu größeren $\mathrm{Hb}$ und $\mathrm{Hk}$ der Kälber bei, wie an den hämatologischen Variablen der Kälber bei 60 LT zu sehen ist (Abb. 2). Die Untersuchungsergebnisse zeigen Zusammenhänge zwischen der Qualität der Erythropoese und deren Auswirkungen über die in den ersten Lebenswochen vorhandene Erythrozytenpopulation und die Ef- 
fekte der Eisenverfügbarkeit während der postnatalen Wachstumsperiode auf hämatologische Variablen und die Gewichtszunahme der Kälber.

\section{Literatur}

ADAMS, R.; GARRY, F.B.; ALDRIDGE, B.; HOLLAND, M.D.; ODDE, K.G.: Hematologic values in newborn beef calves. Am. J. Vet. Res. 53 (1992), 944-950

ADAMS, R.; GARRY, F. B.; ALDRIDGE, B. M.; HOLLAND, M. D.; ODDE, K. G.: Physiologic differences between twin and single born beef calves in the first two days of life. Cornell Vet. 83 (1993), 13-29

BOSTEDT, H.; JEKEL, E.; SCHRAMEL, P.:

Zur Entwicklung der Eisen- und Kupferkonzentration im Blutplasma von Kälbern in den ersten Lebenstagen und -wochen, gleichzeitig ein Beitrag zur larvierten neonatalen Eisenmangelanämie. Dtsch. tierärztl. Wschr. 97 (1990), 400-403

BOSTEDT, H.; HOSPES, R.; WEHREND, A.; SCHRAMEL, P.:

Auswirkungen einer parenteralen Eisenzufuhr auf den Eisenversorgungsstatus in der frühen postnatalen Entwicklungsperiode beim Kalb. Tierärztl. Umschau 22 (2000), 305-315

CAMBIER, C.; CLERBAUX, T.; DETRY, B.; BEERENS, D.; FRANS, A.; GUSTIN, P.: Blood oxygen binding in double-muscled calves and dairy calves with conventional muscle conformation. Am. J. Vet. Res. 61 (2000), 299-304

GUSTIN, P.; DETRY, B.; ROBERT, A.:

Influence of age and breed on the binding of oxygen to red blood cells of bovine calves. J. Appl. Physiol. 82 (1997), 784-790

JEKEL, E.:

Über Konzentrationsänderungen von Elektrolyten und Spurenelementen im Blutplasma von Kälbern in den ersten Lebensstunden und -tagen. Univ. Gießen, Vet. Med. Diss., 1986

KAKUTA, K.; ORINO, K.; YAMAMOTO, S.; WATANABE, K.:

High levels of ferritin and its iron in fetal bovine serum. Comp. Biochem. Physiol. 118A (1997), 165169

KNOWLES, T. G.; EDWARDS, J. E.; BAZELEY, K. J.; BROWN, S. N.; BUTTERWORTH, A.; WARRISS, P. D.:

Changes in the blood biochemical and haematological profile of neonatal calves with age. Veterinary Record 147 (2000), 593-598

KÜHNE, S.; HAMONN, H.M.; BRUCKNER, R.M.; MOREL, C.; ZBINDEN, Y.; BLUM, J.W.: Growth performance, metabolic and endocrine traits, and absorptive capacity in neonatal calves fed either colostrum or milk replacer at two levels. J. Anim. Sci. 78 (2000), 609-620

KUME, S.-I.; TANABE, S.: Effect of parity on colostral mineral concentrations of Holstein cows and value of colostrum as a mineral source for newborn calves. J. Dairy Sci. 76 (1993), 1654-1660

KUME, S.-I.; TANABE; S.:

Effect of supplemental lactoferrin with ferrous iron on iron status of newborn calves. J. Dairy Sci. 79 (1996), 459-464

KURZ, M.M.; WILLETT, L.B.:

Carbohydrate, enzyme, and hematology dynamics in newborn calves. J. Dairy Sci. 74 (1991), 21092118

LIM, G.B.; DODIC, M.; EARNEST, L.; JEYASEELAN, K.; WINTOUR, E.M.: Regulation of erythropoietin gene expression in fetal sheep by glucocorticoids. Endocrinology 137 (1996), 1658-1663

LINDT, F.; BLUM, J. W.:

Occurrence of iron deficiency in growing cattle. J. Vet. Med. A 41 (1994), 237-246

MILTENBURG, G. A. J.; WENSING, T.; van VLIET, J. P. M.; SCHUIJT, G.; van de BROEK, J.; BEUKINK, H. J.:

Blood hemoglobin, plasma iron, and tissue iron in dams in late gestation, at calving, and in veal calves at delivery and later. J. Dairy Sci. 74 (1991), 3086-3094

MIYATA, Y.; FURUGOURI, K.; SHIJIMAYA, K.: Developmental changes in serum ferritin concentration of dairy calves. J. Dairy Sci. 67 (1984), 12561263

NAGASAKO, Y.; SAITO, H.; TAMURA, Y.; SHIMAMURA, S.; TOMITA, M.: Iron-binding properties of bovine lactoferrin in iron-rich solution. J. Dairy Sci. 76 (1993), 1876-1881

SCHÜMANN, K.; HUNDER, G.:

Zur Resorption und Supplementierung von Eisen. Lab. Med. 22 (1998), 465-470 
STEINHARDT, M.; BÜNGER, U.; LANGANKE, M.; GOLLNAST, I.; KUTSCHKE, J.:

Spurenelementausstattung neugeborener Kälber - Einflüsse des Muttertieres sowie von genetischer Konstruktion, Geschlecht und Reifegrad des Neugeborenen. Dtsch. tierärztl. Wschr. 100 (1993), 121 124

STEINHARDT, M.; THIELSCHER, H.-H.; GRÜNBERG, W.:

Tiergerechte Haltung und physiologische Funktionen von Tieren. Entwicklungsqualität und Anpassung von Saugkälbern der Mutterkuhhaltung und von in Gruppen am Tränkeautomaten aufgezogenen Kälbern der Deutschen Rotbunten. Landbauforschung Völkenrode 50 (2000), 181-198

STEINHARDT, M.; THIELSCHER, H.-H.:

Tiergerechte Haltung und physiologische Funktionen von Tieren. Entwicklungsqualität und Anpassung von Kälbern in spezifischen Altersperioden während der Aufzucht in verschiedenen Haltungsvarianten und ihre Beziehung zu metabolischen und hämatologischen Variablen sowie zur Herzschlagfrequenz. Landbauforschung Völkenrode 48 (1998), 118-138

STEINHARDT, M.; THIELSCHER, H.-H.:

Tiergerechte Haltung und physiologische Funktionen von Tieren. Entwicklungsqualität von Milchrindkälbern nach der Geburt und frühe postnatale Adaptation der Tiere in Gruppenhaltung mit Tränkeautomatenfütterung. Tierärztl. Umschau 55 (2000), 189-198

STEINHARDT, M.; THIELSCHER, H.-H.:

Effekte der Entwicklungsqualität auf die Herzfrequenz, die Aktivitäts- und Ruhezeiten und deren Rhythmizität sowie auf die Wachstumsleistung der Milchrindkälber während der Aufzucht in Gruppenhaltung mit Tränkeautomatenfütterung. Arch. Tierz., Dummerstorf 45 (2002) 6, 523-534

TSUJI, S.; HIRATA, Y.; MUKAI, F.:

Comparison of lactoferrin content in colostrum between different breeds. J. Dairy Sci. 73 (1990), 125128

WELCHMAN, D. DE B.; WHELEHAN, O.P.; WEBSTER, A.J.F.:

Haematology of veal calves reared in different husbandry systems and the assessment of iron deficiency. Vet. Rec. 123 (1988), 505-510

WILSON, L.L.; SMITH, J.L.; SMITH, D.L.; SWANSON, D.L.; DRAKE, T.R.; WOLFGANG, D.R.; WHEELER, E.F.:

Characteristics of veal calves upon arrivel, at 28 and 84 days, and at end of the production cycle. J. Dairy Sci. 83 (2000), 843-854

WINTOUR, E. M.; SMITH, M. B.; BELL, R. J.; MCDOUGALL, J. G.; GAUCHI, M. N.:

The role of fetal adrenal hormones in the switch from fetal to adult globin synthesis in the sheep. $\mathrm{J}$. Endocrinol. 104 (1985), 165-170

Eingegangen: 07.10.2002

Akzeptiert: 28.05.2003

Anschrift der Verfasser

Dr. habil. MARTIN STEINHARDT, Dr. HANS - HERMANN THIELSCHER

Bundesforschungsanstalt für Landwirtschaft (FAL)

Institut für Tierschutz und Tierhaltung

D-23847 Westerau 